\title{
Sonothromblysis in Combination with Thrombolytic Drugs in a Rabbit Model Using MRI-Guidance
}

\author{
Christakis Damianou ${ }^{1}$, Nicos Mylonas ${ }^{2}$, Kleanthis Ioannides ${ }^{3}$ \\ ${ }^{1}$ Cyprus University of Technology, Cyprus \\ ${ }^{2}$ Frederick University Cyprus, Limassol, Cyprus \\ ${ }^{3}$ Polikliniki Ygia, Limassol, Cyprus \\ Email: christakis.damianou@cut.ac.cy, bus.mn@fit.ac.cy
}

Received June 2013

\begin{abstract}
The potential of MRI-guided focused ultrasound (MRgFUS) combined with the thrombolytic drug recombinant tissue plasminogen activator (rt-PA), to dissolve clots in the carotid of a New Zealand rabbit in vivo is evaluated. A spherically-focused transducers of $5 \mathrm{~cm}$ diameter; focusing at $10 \mathrm{~cm}$ and operating at $1 \mathrm{MHz}$ was used. A pulsed ultrasound protocol was used that maintains a tissue temperature increase of less than $1^{\circ} \mathrm{C}$ in the clot (called safe temperature). MRgFUS has the potentials to dissolve clots that are injected in the carotid of rabbits in vivo. It was found that the time needed for opening the carotid artery using ultrasound and rt-PA was decreased compared to just using rt-PA. The proposed protocol was monitored using Magnetic Resonance Angiography (MRA) every 1 min.
\end{abstract}

Keywords: Ultrasound; MRI; Stroke; Thrombolysis

\section{Introduction}

The feasibility of ultrasound to enhance thrombolysis was reported in the mid 70s [1,2]. In the following years, several in vitro studies (Kimura et al. 1994 [3] and Spengos K et al. 2000 [4]) have confirmed the above results. In the former studies, the range of intensity varied from $0.2-2.0 \mathrm{~W} / \mathrm{cm}^{2}$ (spatial peak temporal average intensity) and the frequency from $20 \mathrm{kHz}$ to $2 \mathrm{MHz}$ using unfocused ultrasound.

It is speculated that ultrasound accelerates enzymatic fibrinolysis primarily through mechanical mechanisms, by enhancing the effectiveness of thrombolytic drugs, possibly by exposing more binding sites on the fibrin to the recombinant tissue plasminogen activator (rt-PA) [5]. Other mechanical effects of ultrasound, such as cavitation and radiation force, are possibly influencing drug transport $[6,7]$. Acoustic cavitation plays a very significant role in ultrasound-accelerated fibrinolysis [6]. Other theories revolve around the fact that ultrasound promotes motion of fluid around the clot, an effect called microstreaming [7].

Birnbaum et al. in 1998 [8], reported that in vivo arterial clot dissolution can be achieved with intravenous microbubbles and transcutaneous ultrasound delivery alone. Moreover, a study has shown the effectiveness of transcutaneous ultrasound and intravascular microbubbles in lysing intracranial clot in pigs [9].
Administration of gaseous microspheres dramatically lowers the threshold for cavitation and increases the lytic activity of ultrasound (Holland CK and Aplef RE 1990[10]). Because the bubbles are destroyed in the process, they must be constantly injected for complete clot dissolution. Recent studies have demonstrated that microbubbles can be concentrated at the surface of clot by attaching a glycoprotein receptor antagonist, which increases their adherence to acute clot, to the bubbles [11].

The use of Focused ultrasound for destroying clots has received attention lately (Frenkel V et al. 2006 [12]; Shawa G et al. 2009 [13]; Hölscher T et al. 2011[14] and Laing S et al. 2011[15]). In the study by Frenkel V et al. 2006 [12], clots from humans in vitro were ablated using pulse ultrasound with a $1 \mathrm{MHz}$ single element transducer in synergy with rt-PA. In this study the conclusion is that by using rt-PA and focused ultrasound, improved clot destruction rates were achieved. In another study, using unfocused ultrasound at $120 \mathrm{KHz}$ [12], showed that ultrasound has the potential of destroying clots in vitro in combination with Liposomes loaded with rt-PA. Human whole blood clots were ablated though the skull using a hemispheric phased array transducer with 1,000 single piezo elements [13] using pulse ultrasound. The conclusion of this study is that ultrasound alone can be utilized to destroy clots.

In addition to these in vitro studies, there are some in- 
teresting in vivo studies confirming the therapeutic effect of pulse ultrasound (Hölscher T. et al. 2011 [14] and Laing S et al. 2011 [15]). In one study (Laing S et al. 2011 [15]), a rabbit aorta model was used in order to ablate using pulse ultrasound in combination with Liposomes loaded with rt-PA. In another study (Hölscher T. et al. 2011 [14]), clots that were formed in the rabbit marginal ear vein were ablated using pulse ultrasound with a $1 \mathrm{MHz}$ single element transducer in synergy with recombinant tissue plasminogen activator (rt-PA).

Currently there are few but significant clinical trials: a) The CLOTBUST (Combined Lysis of Thrombus in Brain ischemia using transcranial Ultrasound and Systemic Recombinant Tissue-Type Plasminogen Activator (rt$\mathrm{PA})$ ) is a Phase II randomized multi-center international clinical trial [16]; b) The EKOS clinical trial [17] which involves the insertion of a catheter is now being tested in phase II-III Interventional Management of Stroke (IMS) trials; c) The TRUMBI clinical trial (Daffertshofer $\mathrm{M}$ et al. 2005 [18]), using transcranial low-frequency ultrasound-mediated thrombolysis in brain ischemia in combination with intravenous administration of tPA; and d) The TUCSON clinical trial (Barreto A et al. 2009 [19]), a phase I-II randomized placebo-controlled, international multi-center study, using perfultren-lipid microspheres.

In this paper, the therapeutic effect of focused ultrasound and administration of rt-PA to dissolve artificial clot that was injected in the carotid artery of a New Zealand rabbit was examined. In the current study, higher intensities were used than what was proposed in a study by Alexandrov AV et al. in 2004 [20]. We anticipate that by using higher intensities, the rate of clot destruction will be accelerated. The previous studies mostly make use of unfocused ultrasound (Holland CK et al. 2008 [21]; Saguchi T et al. 2008 [22] and Jürgen E et al. 2008 [23]). Thus, the intensity levels that could be used were limited. In this study, focused ultrasound is investigated and therefore higher intensities are utilized provided that thermal effects are avoided. Focused ultrasound targeting the clot is used with spatial average temporal average (SATA) in situ intensity of $20 \mathrm{~W} / \mathrm{cm}^{2}$.

\section{Materials and Methods}

\subsection{HIFU/MRI System}

Figure 1 shows the block diagram of the MRgFUS which includes the following subsystems: 1) Focused ultrasound system, 2) MRI system, 3) Transducer holder and 4) Temperature measurement. Since this system eventually will be utilized in conjunction with MRI the transducer and transducer holder are both designed to be MRI compatible. We have chosen to use MRI, because regarding vascular imaging is considered the gold standard.

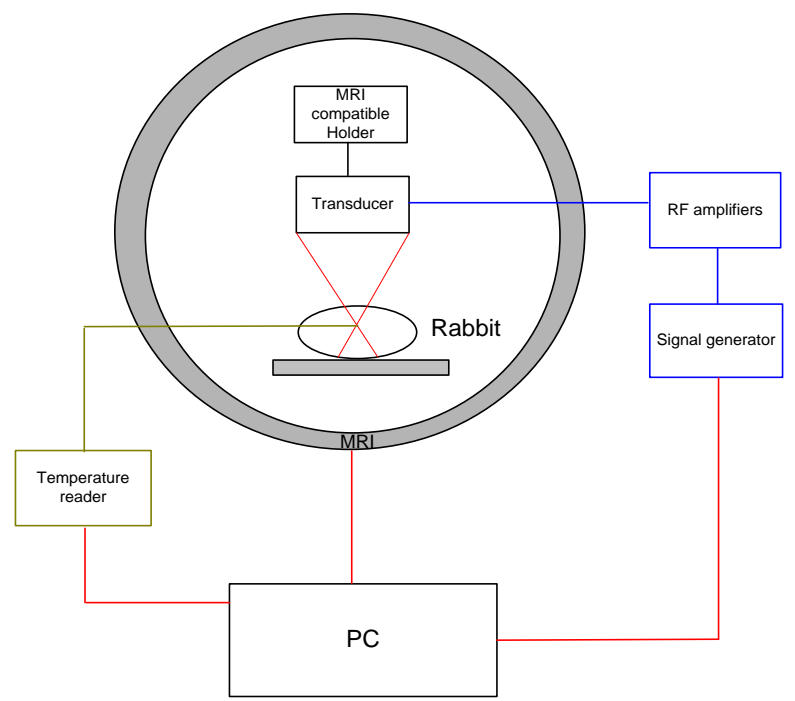

Figure 1. MRgFUS system for in vitro sonothrombolysis.

\subsection{Focused Ultrasound System-MR Imaging}

The ultrasound system consists of a radio frequency (RF) generator/amplifier (1000 W, JJ\&A Instruments, Duvall, WA, USA), and a spherically shaped transducer made from piezoelectric ceramic of low magnetic susceptibility (Piezotechnologies, Etalon, Lebanon, IN, USA). The transducer used operates at $1 \mathrm{MHz}$. The transducer has a focal length of $10 \mathrm{~cm}$ and a diameter of $5 \mathrm{~cm}$. The transducer is coupled to the artery using a special designed plastic holder (MEDSONIC, Limassol, Cyprus). The transducer and transducer holder were placed inside an MRI scanner (Signa 1.5 T, by General Electric, Fairfield, CT, USA).

\subsection{Production of Sample Clots}

Blood clots were obtained by natural coagulation of animal blood samples from healthy cows. The animal experiments protocol was approved by the national body in Cyprus responsible for animal studies (Ministry of Agriculture, Animal Services). Blood was drawn into small containers and placed in a $37^{\circ} \mathrm{C}$ water bath for $3 \mathrm{~h}$ and then stored in a refrigerator at a temperature of $5^{\circ} \mathrm{C}$ for at least $72 \mathrm{~h}$ before use in the experiments to allow complete clot retraction (Holland CK et al. 2008 [21]).

\subsection{Preparation of rt-PA}

The rt-PA was obtained as a lyophilized powder (rt-PA, Actilase, Genentech, San Francisco, CA, USA) mixed with sterile water as per manufacturer's instructions. A dose of $1 \mathrm{mg} / \mathrm{mL}$ was administered.

\subsection{MRI Parameters}

For T2-W FSE the following parameters were used: TR 
$=2500 \mathrm{~ms}$, echo time $(\mathrm{TE})$ was variable from $10 \mathrm{~ms}$ to $160 \mathrm{~ms}$, slice thickness $=3 \mathrm{~mm}$ (gap $0.3 \mathrm{~mm}$ ), matrix $=$ $256 \times 256, \mathrm{FOV}=16 \mathrm{~cm}, \mathrm{NEX}=1$, and $\mathrm{ETL}=8$. For the MRI sequence of MRA the following parameters were used: repetition time (TR) $=40 \mathrm{~ms}$, echo time (TE) $=2.7 \mathrm{~ms}$, Field of View $(\mathrm{FOV})=16 \mathrm{~cm}$, matrix $=256 \times$ 256, flip angle $=10^{\circ}$, Bandwidth $=15.6 \mathrm{KHz}$, Number of excitations $(\mathrm{NEX})=1$. A spinal coil (USA instruments, Cleveland, OH, USA) was used to acquire the MRI signal.

\subsection{In Vivo Experiments}

For the in vivo experiments, 16 New Zealand adult rabbits were used weighting approximately $3.5-4 \mathrm{~kg}$. The rabbits were anaesthetized using a mixture of $500 \mathrm{mg}$ of ketamine (100 mg/mL, Aveco, Ford Dodge, IA), $160 \mathrm{mg}$ of xylazine $(20 \mathrm{mg} / \mathrm{mL}$, Loyd Laboratories, Shenandoah, IA), and $20 \mathrm{mg}$ of acepromazine $(10 \mathrm{mg} / \mathrm{mL}$, Aveco, Ford Dodge, IA) at a dose of $1 \mathrm{~mL} / \mathrm{kg}$. The animal experiments protocol was approved by the national body in Cyprus responsible for animal studies (Ministry of Agriculture, Animal Services).

For the in vivo experiments, the clot was injected in the carotid artery using a thin needle. Once the blockage of the artery was confirmed, ultrasound and rt-PA therapy was applied. A1 $\mathrm{mg} / \mathrm{ml} / \mathrm{kg}$ rt-PA was injected in the jugular vein. Prior to the application of ultrasound a bolus of an ultrasound contrast agent (SonoVue; Bracco $\mathrm{SpA}$, Milan, Italy) was injected intravenously through the ear vein at a dose of $0.02 \mathrm{~mL} / \mathrm{kg}$.

\section{Results}

Figure 2(a) shows the coupling of the transducer to the carotid artery and Figure 2(b) shows the MRI image using $\mathrm{T} 2-\mathrm{W}$ of the coupling of the ultrasonic transducer to the carotid artery of the rabbit. The intense signal indicates the water of the transducer holder which makes an excellent coupling to the rabbit.

Figure 4(a) shows the carotid artery using MRA before the injection of the thrombus. Figure 4(b) shows the MRA image of the carotid artery immediately after the injection of the thrombus. Figure 4(c) shows the MRA image of the carotid artery after applying Ultrasound ( $\mathrm{f}=$ $1 \mathrm{MHz}$, SATA intensity $=20 \mathrm{~W} / \mathrm{cm}^{2}$, Duty factor $=10 \%$, pulse repetition rate $=10 \mathrm{~Hz}$,) and rt-PA for 70 mins (the artery is completely opened).

\section{Discussion}

The results in this study demonstrate the ability of MRgFUS in combination with rt-PA to dissolve clots in an in vivo model. We have proved the capability of the clot model (thrombus) to block the carotid artery and also

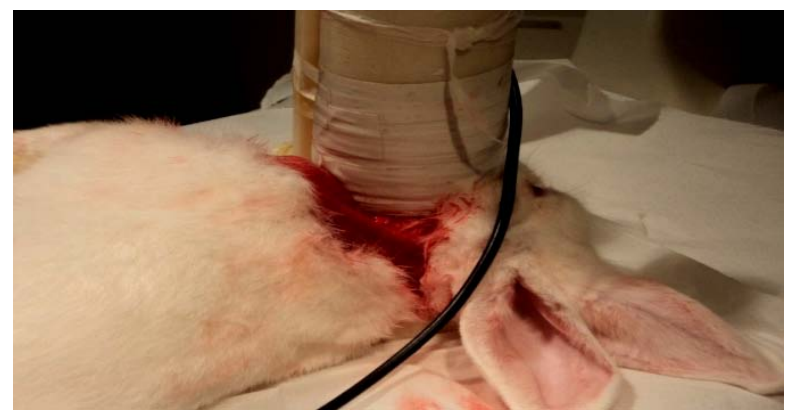

Figure 2. (a). Transducer coupling to the artery (b) MR image using T2-W FSE for the coupling of the ultrasonic transducer to the neck of the rabbit.

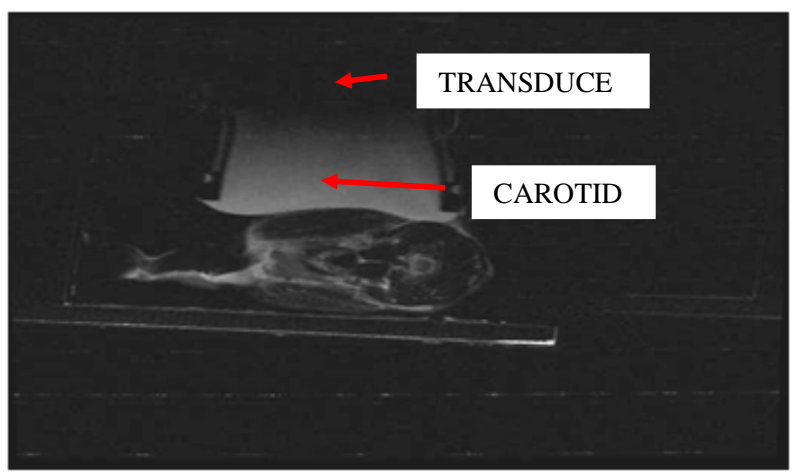

Figure 3. Shows the coupling of the transducer to the carotid artery.

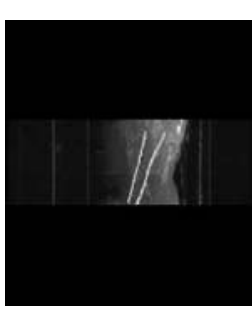

(a)

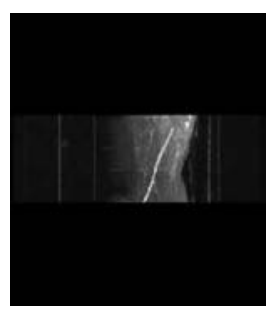

(b)

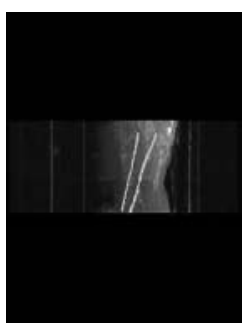

(c)
Figure 4. (a) MRA image of carotid artery before the injection of the thrombus; (b) MRA image of carotid artery immediately after the injection of the thrombus; (c) MRA image of the carotid artery after applying Ultrasound and rt-PA for $70 \mathrm{~min}$.

proved the ability of therapeutic ultrasound in synergy with rt-PA to dissolve clots and at the same time monitoring the whole process using MRA. We have chosen to use the carotid artery because most of the arteries in such a small animal such us the rabbit cannot be visualized with MRI due to spatial resolution problems (for example ear, femoral, middle cerebral artery etc).

The results of this study clearly show that focused ultrasound has the potential to accelerate the treatment of vascular occlusions (potential application could be ischemic stroke) by reducing the treatment time which is crucial for future clinical treatments (ischemic stroke for 
example). By now, the administration of rt-PA is an effective treatment for acute ischemic stroke, but it is associated with an increased risk of intracranial haemorrhage. Therefore, a potential benefit from the application of focused ultrasound is to use lower rt-PA dose without affecting the functionality of the treatment, and ensuring no haemorrhage is caused due to rt-PA.

\section{REFERENCES}

[1] R. Trubestein, H. R. Bernard, F. Etzel, A. Sobbe, A. Cremer and U. Stumpff, "Thrombolysis by Ultrasound," Clinical Science \& Molecular Medicine, Vol. 51, 1976, pp. 697-698.

[2] K. Tachibana and S. Tachibana, "Ultrasonic Vibration for Boosting Fibrinolytic Effects of Urokinase in Vivo," Thrombosis and Haemostasis, Vol. 46, 1981, p. 211.

[3] M. Kimura, S. Iijima, K. Kobayashi and H. Furuhata, "Evaluation of the Thrombolytic Effect of Tissue-Type Plasminogen Activator with Ultrasound Irradiation: In Vitro Experiment Involving Assay of the Fibrin Degradation Products from the Clot,” Biological \& Pharmaceutical Bulletin, Vol. 17, No. 1, 1994, pp. 126-130. http://dx.doi.org/10.1248/bpb.17.126

[4] K. Spengos, S. Behrens, M. Daffertshofer, C. E. Dempfle and M. Hennerici, "Acceleration of Thrombolysis with Ultrasound through the Cranium in a Flow Model," Ultrasound in Medicine \& Biology, Vol. 26, 2000, pp. 889895. http://dx.doi.org/10.1016/S0301-5629(00)00211-8

[5] C. W. Francis, A. Blinc, S. Lee and C. Cox, "Ultrasound Accelerates Transport of Recombinant Tissue Plasminogen Activator into Clots," Ultrasound in Medicine \& Biology, Vol. 21, 1995, pp. 419-424. http://dx.doi.org/10.1016/0301-5629(94)00119-X

[6] A. Blinc, C. W. Francis, J. L. Trudnowski and E. L. Carstensen, "Characterization of Ultrasound-Potentiated Fibrinolysis in Vitro,” Blood, Vol. 81, 1993, pp. 2636-2643.

[7] J. F. Polak, "Ultrasound Energy and the Dissolution of Thrombus,” The New England Journal of Medicine, Vol. 351, 2004, pp. 2154-2155. http://dx.doi.org/10.1056/NEJMp048249

[8] Y. Birnbaum, H. Luo, T. Nagai, M. C. Fishbein, T. M. Peterson, S. Li, D. Kricsfeld, T. R. Porter and R. J. Siegel, "Noninvasive in Vivo Clot Dissolution without a Thrombolytic Drug: Recanalization of Thrombosed Iliofemoral Arteries by Transcutaneous Ultrasound Combined with Intravenous Infusion of Microbubbles," Circulation, Vol. 97, 1998, pp. 130-134.

http://dx.doi.org/10.1161/01.CIR.97.2.130

[9] W. C. Culp, E. Erdem, P. K. Roberson and M. M. Husain "Microbubble Potentiated Ultrasound as a Method of Stroke Therapy in a Pig Model: Preliminary Findings," Journal of Vascular and Interventional Radiology, Vol. 14, No. 11, 2003, pp. 1433-1436. http://dx.doi.org/10.1097/01.RVI.0000096767.47047.FA

[10] C. K. Holland and R. E. Apfel, "Thresholds for Transient Cavitation Produced by Pulsed Ultrasound in a Controlled Nuclei Environment," Journal of the Acoustical Society of
America, Vol. 88, No. 5, 1990, pp. 2059-2069. http://dx.doi.org/10.1121/1.400102

[11] P. A. Schumann, J. P. Christiansen, R. M. Quigley, T. P. McCreery, R. H. Sweitzer and E. C. Unger, "Targeted-Microbubble Binding Selectively to GPIIb IIIa Receptors of Platelet Thrombi," Investigative Radiology, Vol. 37, No. 11, 2002, pp. 587-593. http://dx.doi.org/10.1097/00004424-200211000-00001

[12] V. Frenkel, J. Oberoi, M. Stone, M. Park, C. Deng, B. Wood, Z. Neeman, M. Horne III and K. Li, "Pulsed High-Intensity Focused Ultrasound Enhances Thrombolysis in an in Vitro Model,” Radiology, Vol. 239, No. 1, 2006, pp. 86-93. http://dx.doi.org/10.1148/radiol.2391042181

[13] G. Shawa, J. Meunier, S. Huang, C. Lindsell, D. McPherson, C. Holland, "Ultrasound-Enhanced Thrombolysis with tPA-Loaded Echogenic Liposomes," Thrombosis Research, Vol. 124, No. 3, 2009, pp. 306-310. http://dx.doi.org/10.1016/j.thromres.2009.01.008

[14] T. Hölscher, D. Fisher, R. Raman, K. Ernstrom, E. Zadicario, W. Bradley and A. Voie, "Noninvasive Transcranial Clot Lysis Using High Intensity Focused Ultrasound," Journal of Neurology \& Neurophysiology, Vol. 1, 2011, pp. 1-6.

[15] S. Laing, M. Moody, B. Smulevitz, H. Kim, P. Kee, S. Huang, C. Holland and D. McPherson, "Ultrasound-Enhanced Thrombolytic Effect of Tissue Plasminogen Activator-Loaded Echogenic Liposomes in an in Vivo Rabbit Aorta Thrombus Model-Brief Report," Arteriosclerosis, Thrombosis, and Vascular Biology, Vol. 31, No. 6, 2011, pp. 1357-1359. http://dx.doi.org/10.1161/ATVBAHA.111.225938

[16] A. V. Alexandrov, A. W. Wojner and J. C. Grotta, "Clotbust Investigators Clotbust: Design of a Randomized Trial of Ultrasound-Enhanced Thrombolysis for Acute Ischemic Stroke,” Journal of Neuroimaging, Vol. 14, 2004, pp. 108-12

[17] The IMS Study Investigators, "Combined Intravenous and Intraarterial Recanalization for Acute Ischemic Stroke: The Interventional Management of Stroke Study,” Stroke, Vol. 35, 2004, pp. 904-912. http://dx.doi.org/10.1161/01.STR.0000121641.77121.98

[18] M. Daffertshofer, A. Gass and P. Ringleb, "Transcranial Low Frequency Ultrasound-Mediated Thrombolysis in Brain Ischemia: Increased Risk of Hemorrhage with Combined Ultrasound and Tissue Plasminogen Activator," Stroke, Vol. 36, 2005, pp. 1441-1446. http://dx.doi.org/10.1161/01.STR.0000170707.86793.1a

[19] A. Barreto, V. Sharma, A. Lao, P. Schellinger, P. Amarenco, P. Sierzenski, A. Alexandrov and C. Molina, "Safety and Dose-Escalation Study Design of Transcranial Ultrasound in Clinical SONolysis for Acute Ischemic Stroke: the Tucson Trial," International Journal of Stroke, Vol. 4, 2009, pp. 42-48. http://dx.doi.org/10.1111/j.1747-4949.2009.00252.x

[20] A. V. Alexandrov, C. A. Molina and J. C. Grotta "Ultrasound Enhanced Systemic Thrombolysis for Acute Ischemic Stroke,” The New England Journal of Medicine, Vol. 351, 2004, pp. 2170-2178. 
http://dx.doi.org/10.1056/NEJMoa041175

[21] C. K. Holland, S. S Vaidya, S. Datta, C. C. Coussios and G. J. Shaw, "Ultrasound Enhanced Tissue Plasminogen Activator Thrombolysis in an in Vitro Porcine Clot Model,” Thrombosis Research, Vol. 121, 2008, pp. 663-673. http://dx.doi.org/10.1016/j.thromres.2007.07.006

[22] T. Saguchi, H. Onoue, M. Urashima, T. Ishibashi, T. Abe and H. Furuhata, "Effective and Safe Conditions of Low-Frequency Transcranial Ultrasonic Thrombolysis for Acute Ischaemic Stroke: Neurologic and Histologic Evaluation in a Rat Middle Cerebral Artery Stroke Model,”
Stroke, Vol. 39, 2008, pp. 1007-1011. http://dx.doi.org/10.1161/STROKEAHA.107.496117

[23] E. Jürgen, R. K. Inke, K. Björn, H. Götz and S. Günter, "Sonothrombolysis with Transcranial Color-Coded Sonography and Recombinant Tissue-Type Plasminogen Activator in Acute Middle Cerebral Artery Main Stem Occlusion: Results from a Randomized Study,” Stroke, Vol. 39, 2008, pp. 1470-1475. http://dx.doi.org/10.1161/STROKEAHA.107.503870 